\title{
Effects of crack surface electrostatic tractions on the fracture behaviour of
}

\section{magnetoelectric composite materials}

\author{
Aibing Zhang ${ }^{\mathrm{a}, *}$, Baolin Wang ${ }^{\mathrm{b}}$ \\ a Piezoelectric Device Laboratory, School of Mechanical Engineering \& Mechanics, Ningbo \\ University, Ningbo, 315211, P.R. China \\ b Institute for Infrastructure Engineering, Western Sydney University, Penrith, NSW 2751,
} Australia

\begin{abstract}
The fracture mechanics problem of a Griffith crack embedded in a two-dimensional magnetoelectric composite material subjected to coupling mechanical, electric and magnetic loads at infinity is investigated. The crack surfaces electrostatic tractions are taken into account and the crack is assumed to be electrically and magnetically semi-permeable. Explicit and closed form solutions of electric displacement and magnetic induction inside the crack, stress, electric displacement and magnetic induction intensity factors are derived based on the extended Stroh formalism and continuous distribution of generalized dislocation approach. Numerical computations are also carried out to illustrate the influence of electrostatic tractions on the crack tip field when the crack interior is filled with different dielectric medium. It is found that the electrostatic tractions on the crack surfaces have the tendency to close the crack thus retard the crack propagation, and the traditional traction-free crack model always overestimates the effect of applied magnetoelectric loads on the crack tip filed intensity factors. Crack surfaces electrostatic tractions cannot be neglected for large applied electric or magnetic load to mechanical load ratio, and small dielectric constant and magnetic permeability of the crack interior.
\end{abstract}

* Corresponding author. Tel.: +86 75487600980

E-mail address: zhangaibing@ nbu.edu.cn (Aibing Zhang). 
Keywords: Magnetoelectric; Electrostatic tractions; Boundary condtions; Crack

\section{Introduction}

Magnetoelectric composite materials made of piezoelectric and piezomagnetic phase have been found extensively applications in modern industries such as sensors, actuators, transducers, hydrophones, electric packaging and acoustic/ultrasonic devices due to coupling mechanical, electric and magnetic effect (Alshits et al., 1992; Huang and Kuo, 1997; Nan, 1994). However, because of their brittleness and low strength, cracks and flaws in such composites inevitably occur either during manufacturing process or when subjected to mechanical, electric and magnetic loads. Thus, fracture mechanics problems in magnetoelectric composite materials have been received tremendous interest. Green's functions for an infinite two-dimensional magnetoelectroelastic medium containing an elliptical cavity or a crack were derived based on the extended Stroh formalism and complex function method by Liu et al. (2001). Crack initiation behavior in magnetoelectric composite materials under in-plane deformation was studied by Song and Sih (2003). The interaction problem of multiple arbitrarily oriented and distributed cracks in magnetoelectroelastic materials was considered by Tian and Gabbert (2004). Green's functions for the various defects embedded in an infinite magnetoelectroelastic material under thermal loading were obtained with the framework of in-plane magnetoelectroelastic interactions by Qin (2005). García-Sánchez et al. (2007) analysed that the behavior of cracked linear magnetoelectroelastic materials by using the dual boundary element method approach. Recently, to eliminate the singularity behavior on the crack tips, a pre-fracture zone on electrically impermeable and magnetically permeable interface crack model between two dissimilar magnetoelectroelastic materials was developed by $\mathrm{Ma}$ et al. 
(2013). The solutions for electrically and magnetically impermeable crack branching out of the crack plane in a magnetoelectric composite materials under thermo-electro-magneto-mechanical loads were presented by Zhang and Wang (2014a). Hu et al. (2015) investigated interface crack problem by extending the concept of Dugdale crack model and Yoffe model to a moving crack between two dissimilar magnetoelectroelastic materials. For more contributions in the literature on the fracture mechanics of magnetoelectric composite materials, see Wang and Shen (2002), Gao et al. (2003), Sih and Song (2003), Qin (2004), Soh and Liu (2005), Ootao and Tanigawa (2005), Tupholme (2009), Rojas-Díaz et al. (2012), Hu et al. (2014), Sladek et al. (2015), Bhargava et al. (2015), Rogowski (2015) and Ma et al. (2015).

In above-mentioned works, four different boundary conditions on the crack surfaces, there are electrically impermeable and magnetically permeable, electrically permeable and magnetically impermeable, electrically and magnetically impermeable and electrically and magnetically permeable are assumed to investigate the fracture mechanics problem in magnetoelectric composites. Wang and Mai (2007) discussed the applicability of the crack-face electromagnetic boundary conditions, and pointed out that the electric and magnetic permeabilities of air or vacuum inside the crack cannot be ignored. Taking electromagnetic properties of crack interior into consideration and using magnetoelectric boundary conditions at the crack face related to crack opening displacement, the fracture mechanics problem of magnetoeletroelastic solid was solved by Zhong and Li (2007). To date, the crack surfaces stress boundary conditions of most magnetoelectric composites crack problems are still considered to be free, which is identical with the typical assumption for the fracture mechanics analysis of purely elastic materials. Generally, an opened 
crack filled with air or vacuum represents an obstacle against an electric field perpendicular to the crack plane, since the dielectric constant of air or vacuum is at least three orders of magnitude smaller than that of magnetoelectric composites. Electric field that passes the crack gap causes a normal electric potential jump across the crack and induces electrostatic stresses that pull the crack surfaces together (McMeeking and Landis, 2005; Neumeister et al., 2013). The electrostatic tractions acting upon crack faces should be introduced in piezoelectric medium fracture evaluation together with the electrically semi-permeable crack model, especially when the mechanical loads is not too large and the electric displacement loads is not too small (Ricoeur and Kuna, 2009). The axisymmetric problem of a penny-shaped crack embedded in an infinite three-dimensional piezoelectric body is considered by $\mathrm{Li}$ et al. (2011). The effect of electrostatic tractions on the fracture behavior of a piezoelectric material under mechanical and/or electric loading is analyzed by Xie et al. (2014) and Zhang and Wang (2014b).

However, in contrast to the piezoelectric materials, very few papers can be found on the influence of crack surfaces electrostatic tractions on magnetoelectric composite materials fracture mechanics problem. On the other hand, due to the pronounced piezoelectric, piezomagnetic and magnetoelectric properties, the electric displacement on the crack surfaces does not vanish and has a non-negligible magnitude even if only a mechanical (or magnetic induction) loads is applied at infinity to a magnetoelectric composite material with a crack filled with air/vacuum. Therefore, the primary objective of this paper is to investigate effect of crack surfaces electrostatic tractions on the fracture behaviour of magnetoelectric composites. The plan of the paper is as follows. The extended Stroh formalism is outlined in Section 2. In Section 3 the explicit solutions of stress, electric displacement and magnetic induction intensity 
factors are derived for magnetoelectric composite materials problem. Some numerical results are presented in Section 4, and concluding remarks are made in Section 5.

\section{The Stroh formalism}

Consider a linear magnetoelectroelstic material in which all fields are assumed to depend only on the in-plane coordinates $x_{1}$ and $x_{2}$. The basic equations (constitutive, divergence and gradient) of the linear magnetoelectric composite solids without any body force, electric charge and electric current are (Liu et al., 2001):

$$
\begin{gathered}
\sigma_{i j}=c_{i j k l} u_{k, l}-e_{l i j} \phi_{, l}-h_{l i j} \varphi_{, l} \\
D_{i}=e_{i k l} u_{k, l}+\kappa_{i l} \phi_{, l}+\alpha_{i l} \varphi_{, l} \\
B_{i}=h_{i k l} u_{k, l}+\alpha_{i l} \phi_{, l}+\mu_{i l} \varphi_{, l} \\
\sigma_{i j, i}=0, D_{i, i}=0, B_{i, i}=0 \\
\varepsilon_{i j}=\frac{1}{2}\left(u_{i, j}+u_{j, i}\right), \quad E_{i}=-\phi_{, i}, H_{i}=-\varphi_{, i}
\end{gathered}
$$

In Eqs. (1)-(3), a comma denotes partial differentiation and repeated indices mean summation. $\sigma_{i j}, \varepsilon_{i j}, D_{i}, E_{i}, B_{i}$ and $H_{i}$ are elastic stress, strain, electric displacement, electric field, magnetic induction and magnetic field, respectively. $u_{i}$, $\phi$ and $\varphi$ are elastic displacement, electric potential and magnetic potential, respectively. $c_{i j k l}, e_{i k l}, h_{i k l}, \alpha_{i j}, \kappa_{i j}$ and $\mu_{i j}$ are the elastic moduli, piezoelectric coefficients, peizoemagnetic coefficients, magnetoelectric coefficients, dielectric constants and magnetic permeability coefficients, respectively.

For two-dimensional deformations, the general solution to the Eq. (1) can be written as

$$
\mathbf{u}=\mathbf{A f}(z)+\overline{\mathbf{A f}(z)}
$$

where $\mathbf{u}=\left[u_{1}, u_{2}, u_{3}, \phi, \varphi\right]^{\mathrm{T}}, \mathbf{f}(z)=\operatorname{diag}\left[f\left(z_{1}\right), f\left(z_{2}\right), f\left(z_{3}\right), f\left(z_{4}\right), f\left(z_{5}\right)\right]$ is arbitrary 
analytic function, $z_{i}=x_{1}+p_{i} x_{2}$, the superscript " $\mathrm{T}$ " and the overbars stand for transposition of a matrix and complex conjugation, respectively. $p_{i}$ and $\mathbf{A}$ are constants determined by

$$
\left[\mathbf{Q}+p_{i}\left(\mathbf{R}+\mathbf{R}^{\mathbf{T}}\right)+p_{i}^{2} \mathbf{T}\right] \mathrm{A}_{i}=0
$$

in which $\mathbf{Q}, \mathbf{R}$, and $\mathbf{T}$ are defined by

$$
\mathbf{Q}=\left[\begin{array}{ccc}
c_{1 k k 1} & e_{1 j 1} & h_{1 j 1} \\
e_{1 k 1}^{T} & -\kappa_{11} & -\alpha_{11} \\
h_{1 k 1}^{T} & -\alpha_{11} & -\mu_{11}
\end{array}\right], \quad \mathbf{R}=\left[\begin{array}{ccc}
c_{1 i k 2} & e_{2 j 1} & h_{2 j 1} \\
e_{1 k 2}^{T} & -\kappa_{12} & -\alpha_{12} \\
h_{1 k 2}^{T} & -\alpha_{12} & -\mu_{12}
\end{array}\right], \quad \mathbf{T}=\left[\begin{array}{ccc}
c_{2 i k 1} & e_{2 j 2} & h_{2 j 2} \\
e_{2 k 2}^{T} & -\kappa_{22} & -\alpha_{22} \\
h_{2 k 2}^{T} & -\alpha_{22} & -\mu_{22}
\end{array}\right]
$$

The stress, electric displacement and magnetic induction obtained by inserting Eqs. (3) and (4) into Eq. (1) can be expressed in terms of the generalized stress function vector $\boldsymbol{\Phi}$ as

$$
\begin{aligned}
& \mathbf{t}_{1}=\left[\sigma_{11}, \sigma_{12}, \sigma_{13}, D_{1}, B_{1}\right]^{\mathrm{T}}=-\mathbf{\Phi}_{, 2} \\
& \mathbf{t}_{2}=\left[\sigma_{21}, \sigma_{22}, \sigma_{23}, D_{2}, B_{2}\right]^{\mathrm{T}}=\boldsymbol{\Phi}_{, 1}
\end{aligned}
$$

where

$$
\begin{aligned}
& \mathbf{\Phi}=\mathbf{B f}(z)+\overline{\mathbf{B f}(z)} \\
& \mathbf{B}=\mathbf{R}^{\mathrm{T}} \mathbf{A}+\mathbf{T A P} \\
& \mathbf{P}=\operatorname{diag}\left[p_{1}, p_{2}, p_{3}, p_{4}, p_{5}\right]
\end{aligned}
$$

\section{Fracture mechanics solution of magnetoeletric composite materials by} considering crack surface electrostatic tractions

Consider a generalized 2D magnetoelectric composite material problem of a single crack of length $2 a$ and filled with air or vacuum, as shown in Fig. 1. The opening displacement of the crack is always very small, the electric displacement and magnetic induction through crack should not be zero. Therefore, it is more reasonable 
to consider the electric and magnetic fields inside the crack and the electric and magnetic potential jumps across the crack surfaces simultaneously, that is magnetoelectric semi-permeable crack faces boundary condition. The remote loading system is composed of stresses $\sigma_{21}^{\infty}, \sigma_{22}^{\infty}$ and $\sigma_{23}^{\infty}$, electric displacement $D_{2}^{\infty}$ and magnetic induction $B_{2}^{\infty}$, i.e.,

$$
\mathbf{t}_{2}^{\infty}=\left[\sigma_{21}^{\infty}, \sigma_{22}^{\infty}, \sigma_{23}^{\infty}, D_{2}^{\infty}, B_{2}^{\infty}\right]^{\mathrm{T}}
$$

The faces of a non-conducting crack in a dielectric material are interfaces between a solid and air, vacuum or any other insulation matter. If either an electric loading is imposed or the material exhibits physical properties coupling magnetical/mechanical and electrical fields, such as piezoelectrics and magnetoelectrics, electrostatic tractions and related displacements are induced at the crack faces. Even a comparatively small crack opening stress induces a crack tip loading which might not be small compared to the low fracture toughness of ceramic materials. Therefore, electrostatic tractions are very essential for assessment of strength for piezoelectric or magnetoelectric materials, and they acting on the crack surfaces have a following form (Ricoeru and Kuna, 2009)

$$
\sigma_{22}^{c}=\frac{1}{2} \frac{\left(D_{2}^{c}\right)^{2}}{\kappa_{c}}\left(1-\frac{\kappa_{c}}{\kappa_{22}}\right)
$$

where $\kappa_{c}$ is the permittivity of air, vacuum or others dielectric medium filling the crack, $D_{2}^{c}$ is the electric displacement inside the crack to be determined, calculated form the capacitor analogy model (Hao and Shen, 1994)

$$
D_{2}^{c}=-\kappa_{c} \frac{\varphi^{+}-\varphi^{-}}{u_{2}^{+}-u_{2}^{-}}
$$

The superscript "+" and "-" refer to the upper and lower main crack surfaces, respectively. 
It is noted that the crack may be breakdown electrically by the mechanism of corona discharges under the applied mechanical and electrical loadings if the dielectric constant of the magnetoelectric composite is much higher than that of the crack interior. In this case $\left|D_{2}^{c}\right| \geq \kappa_{c} E_{d}$ (where $E_{d}$ is the critical electric field level for discharge), the electrostatic tractions acting on the crack surfaces and electric displacement inside the crack are given by Landis (2004) from the viewpoint of energetically equivalent as

$$
\begin{gathered}
\sigma_{22}^{c}=E_{d}\left|\omega_{d}\right|+\frac{1}{2} \kappa_{c} E_{d}^{2} \\
D_{2}^{c}=\kappa_{c} \operatorname{sgn}\left(D_{2}^{c}\right) E_{d}+\omega_{d}
\end{gathered}
$$

where $\omega_{d}$ represents the amount of charge per unit area transferred between the crack surfaces. The purpose of this paper is to exam the effect of crack surface electrostatic tractions on the fracture behaviour of magnetoelectric composite materials for the case of $\left|D_{2}^{c}\right| \leq \kappa_{c} E_{d}$. Electrical breakdown of the crack is out of the scope of this research. Therefore the phenomenon of electrical discharge will not be taken into consideration in this paper.

In order to consider the magnetic effect of the medium inside the crack, it is more realistic to adopt the opening crack model (Gao et al., 2004)

$$
B_{2}^{c}=-\mu_{c} \frac{\varphi^{+}-\varphi^{-}}{u_{2}^{+}-u_{2}^{-}}
$$

In Eq. (14), $B_{2}^{c}$ denotes the magnetic induction normal to the crack faces, and $\mu_{c}$ stands for the magnetic permeability of the medium inside the crack.

By the superposition principle, the crack surfaces boundary conditions for this problem can be expressed as

$$
\begin{aligned}
& \mathbf{t}_{2}\left(x_{1}, x_{2}\right)=-\left[\mathbf{t}_{2}^{\infty}-\mathbf{t}_{2}^{c}\right], \quad\left|x_{1}\right| \leq a, x_{2}=0 \\
& \mathbf{t}_{1}=\mathbf{t}_{2}=0, \quad \text { at infinity }
\end{aligned}
$$


where $\mathbf{t}_{2}^{c}=\left[0, \sigma_{22}^{c}, 0, D_{2}^{c}, B_{2}^{c}\right]^{\mathrm{T}}$.

A magnetoelectroelastic crack can be constructed by continuous distribution of generalized dislocation (i.e., a mechanical dislocation, an electric dipole and a magnetic dipole at the same point) technique with appropriate weight function. The solution of single generalized dislocation in magnetoelectric composites has the similar expression with that in anisotropic materials obtained by Suo (1990). Stress, electric displacement and magnetic induction fields on the plane $x_{2}=0$ of a generalized dislocation located at coordinate origin have the following form

$$
\mathbf{t}_{2}\left(x_{1}, 0\right)=\frac{1}{\pi} \mathbf{d}\left(\frac{1}{x_{1}}\right)
$$

where $\mathbf{Y}=\mathbf{L}+\overline{\mathbf{L}}, \quad \mathbf{L}=i \mathbf{A B}^{-1}, \quad \mathbf{b}=\mathbf{Y d}$ and $\mathbf{b}=\left[b_{1}, b_{2}, b_{3}, b_{4}, b_{5}\right]^{T}$ is the Burgers vector. Enforcing the satisfaction of the boundary conditions (15) on the crack surfaces, a singular integral equation is obtained by distributing generalized dislocation of the preceding form with weight $\mathbf{d}(\xi)$ in the interval $\left|x_{1}\right| \leq a$ as

$$
\int_{-a}^{a} \frac{\mathbf{d}(\xi)}{\pi\left(x_{1}-\xi\right)} d \xi+\mathbf{t}_{2}^{\infty}-\mathbf{t}_{2}^{c}=0,\left|x_{1}\right|<a
$$

The single value condition of the displacement, electric potential and magnetic potential around a closed contour surrounding the whole crack requires that

$$
\int_{-a}^{a} \mathbf{d}(\xi) d \xi=0,\left|x_{1}\right|<a
$$

Eqs. (17) and (18) have well known solution

$$
\mathbf{d}(\xi)=\left[\mathbf{t}_{2}^{\infty}-\mathbf{t}_{2}^{c}\right] \frac{\xi}{\sqrt{a^{2}-\xi^{2}}},|\xi \leq a|
$$

Then the crack opening displacement, the jump of electric potential and magnetic potential can be obtained

$$
\Delta \mathbf{u}=\int_{x_{1}}^{a} \mathbf{b}(\xi) d \xi=\mathbf{Y}\left[\mathbf{t}_{2}^{\infty}-\mathbf{t}_{2}^{c}\right] \sqrt{a^{2}-x_{1}^{2}}
$$


From Eqs. (11) and (14), we have

$$
\begin{aligned}
& D_{2}^{c}=-\kappa_{c} \frac{Y_{42}\left(\sigma_{22}^{\infty}-\sigma_{22}^{c}\right)+Y_{44}\left(D_{2}^{\infty}-D_{2}^{c}\right)+Y_{45}\left(B_{2}^{\infty}-B_{2}^{c}\right)}{Y_{22}\left(\sigma_{22}^{\infty}-\sigma_{22}^{c}\right)+Y_{24}\left(D_{2}^{\infty}-D_{2}^{c}\right)+Y_{25}\left(B_{2}^{\infty}-B_{2}^{c}\right)} \\
& B_{2}^{c}=-\mu_{c} \frac{Y_{52}\left(\sigma_{22}^{\infty}-\sigma_{22}^{c}\right)+Y_{54}\left(D_{2}^{\infty}-D_{2}^{c}\right)+Y_{55}\left(B_{2}^{\infty}-B_{2}^{c}\right)}{Y_{22}\left(\sigma_{22}^{\infty}-\sigma_{22}^{c}\right)+Y_{24}\left(D_{2}^{\infty}-D_{2}^{c}\right)+Y_{25}\left(B_{2}^{\infty}-B_{2}^{c}\right)}
\end{aligned}
$$

Eq. (21) can be reduced to

$$
\alpha_{3}\left(D_{2}^{c}\right)^{3}+\alpha_{2}\left(D_{2}^{c}\right)^{2}+\alpha_{1} D_{2}^{c}+\alpha_{0}=0
$$

with

$$
\begin{aligned}
& \alpha_{3}=\frac{Y_{22}}{2 \kappa_{c}}\left(1-\frac{\kappa_{c}}{\kappa_{22}}\right), \alpha_{2}=Y_{24}+\frac{Y_{42}}{2}\left(1-\frac{\kappa_{c}}{\kappa_{22}}\right), \alpha_{1}=-\left[Y_{22} \sigma_{22}^{\infty}+Y_{24} D_{2}^{\infty}+Y_{25}\left(Y_{24} D_{2}^{\infty}-Y_{24} B_{2}^{c}\right)-\kappa_{c} Y_{44}\right], \\
& \alpha_{0}=-\kappa_{c}\left[Y_{42} \sigma_{22}^{\infty}+Y_{44} D_{2}^{\infty}+Y_{45}\left(B_{2}^{\infty}-B_{2}^{c}\right)\right] .
\end{aligned}
$$

Similarly, Eq. (22) can be reduced to

$$
\beta_{2}\left(B_{2}^{c}\right)^{2}+\beta_{1} B_{2}^{c}+\beta_{0}=0
$$

with

$$
\begin{aligned}
& \beta_{2}=Y_{25}, \beta_{1}=-\left\{Y_{22}\left[\sigma_{22}^{\infty}-\frac{\left(D_{2}^{c}\right)^{2}}{2 \kappa_{c}}\left(1-\frac{\kappa_{c}}{\kappa_{22}}\right)\right]+Y_{24}\left(D_{2}^{\infty}-D_{2}^{c}\right)+Y_{25} B_{2}^{\infty}\right\}, \\
& \beta_{0}=-\mu_{c}\left\{Y_{52}\left[\sigma_{22}^{\infty}-\frac{\left(D_{2}^{c}\right)^{2}}{2 \kappa_{c}}\left(1-\frac{\kappa_{c}}{\kappa_{22}}\right)\right]+Y_{54}\left(D_{2}^{\infty}-D_{2}^{c}\right)+Y_{55} B_{2}^{\infty}\right\} .
\end{aligned}
$$

Eq. (23) is a cubic equation and its general solution can be found from mathematics handbook. For reasonable material constants and loading conditions, Eq. (23) has three roots, but only one root is physically acceptable. In this paper, the solution has the following form:

$$
D_{2}^{c}=-\frac{\alpha_{2}}{3 \alpha_{3}}+\frac{-1-\sqrt{3} i}{2} \sqrt[3]{P+\sqrt{P^{2}+Q^{3}}}+\frac{-1+\sqrt{3} i}{2} \sqrt[3]{P-\sqrt{P^{2}+Q^{3}}}
$$

where $P$ and $Q$ are two known real constants depending on the applied loads, the 
material constants, dielectric constants and the magnetic permeability of the medium inside the crack as formulated below:

$$
P=\frac{\alpha_{2} \alpha_{1}}{6 \alpha_{3}^{2}}-\frac{\alpha_{2}^{3}}{27 \alpha_{3}^{3}}-\frac{\alpha_{0}}{2 \alpha_{3}}, \quad Q=\frac{\alpha_{1}}{3 \alpha_{3}}-\frac{\alpha_{2}^{2}}{9 \alpha_{3}^{2}}
$$

It follows from Eq. (24) that the magnetic induction at the crack surfaces is

$$
B_{2}^{c}=-\frac{\beta_{1}+\sqrt{\beta_{1}^{2}-4 \beta_{2} \beta_{0}}}{2 \beta_{2}}
$$

The stress, electric displacement and magnetic induction field on the plane $x_{2}=0$ are obtained by Eqs. (17) and (19)

$$
\mathbf{t}_{2}\left(x_{1}, 0\right)=\left[\mathbf{t}_{2}^{\infty}-\mathbf{t}_{2}^{c}\right]\left[\frac{x_{1}}{\sqrt{x_{1}^{2}-a^{2}}}-1\right]
$$

Defining the field intensity factor vector

$$
\mathbf{K}=\left[K_{I I}, K_{I}, K_{I I I}, K_{D}, K_{B}\right]^{T}=\lim _{x_{1} \rightarrow a^{+}} \sqrt{2 \pi\left(x_{1}-a\right)} \mathbf{t}_{2}
$$

Inserting Eq. (29) into Eq. (28) gives

$$
\mathbf{K}=\left[\mathbf{t}_{2}^{\infty}-\mathbf{t}_{2}^{c}\right] \sqrt{\pi a}
$$

\section{Numerical results and discussion}

Magnetoelectric coupling effect has been observed in single-phase multiferroic material at low temperature where simultaneous electric and magnetic ordering coexists, and in two phase composites where the participating phases are piezoelectric and piezomagnetic. Although over ten different compound families have been widely studied as multiferroic magnetoelectric materials, a high inherent coupling (especially above room temperature) has not yet been found in the single-phase compound, which hinders their applications (Nan et al., 2008). However, magnetoelectric composites made by combining piezoelectric and magnetic substances together can produce a 
large magnetoelectric response at the room temperature (Nan, 1994). The magnetoelectric effect in composite materials is result of the product of the mechanical/electrical effect in the piezoelectric phase and the magnetic/mechanical effect in the piezomagnetic one. This is a coupled electrical and magnetic phenomenon via elastic interaction. It is noted that the applied constants of magnetoelectric material are controversy in the literature, and a complete set of material properties of single-phase multiferroic materials, such as $\mathrm{BiFeO}_{3}, \mathrm{YMnO}_{3}$, $\mathrm{Cr}_{2} \mathrm{O}_{3}, \mathrm{LuFe}_{2} \mathrm{O}_{3}$, etc., cannot be found. In this paper, the magnetoelectric composite material is made of piezoelectric phase $\mathrm{BaTiO}_{3}$ and magnetostrictive phase $\mathrm{CoFe}_{2} \mathrm{O}_{4}$, and the ratio of $\mathrm{BaTiO}_{3}$ to $\mathrm{CoFe}_{2} \mathrm{O}_{4}$ is roughly $0.5: 0.5$. Hence, the fundamental purpose of this paper is to present an analytical method to investigate the influence of crack surfaces electrostatic tractions on crack tip fields in the magnetoelectric medium. The variation tendency of intensity factors under applied magnetoelectroelastic loading is correct although the material properties may not be accuracy enough. The material constants are taken as (Sih and Chen, 2003),

Elastic constants:

$c_{11}=226 \mathrm{GPa}, c_{13}=124 \mathrm{GPa}, c_{33}=216 \mathrm{GPa}, c_{44}=44 \mathrm{GPa}$

Piezoelectric constants:

$$
e_{31}=-2.2 \mathrm{C} / \mathrm{m}^{2}, e_{33}=9.3 \mathrm{C} / \mathrm{m}^{2}, e_{15}=5.8 \mathrm{C} / \mathrm{m}^{2}
$$

Dielectric constants:

$$
\kappa_{11}=56.4 \times 10^{-10} \mathrm{C}^{2} / \mathrm{Nm}^{2}, \quad \kappa_{33}=63.5 \times 10^{-10} \mathrm{C}^{2} / \mathrm{Nm}^{2}
$$

Piezomagnetic constants:

$$
h_{31}=290.2 \mathrm{~N} / \mathrm{Am}, h_{33}=350 \mathrm{~N} / \mathrm{Am}, h_{15}=275 \mathrm{~N} / \mathrm{Am}
$$

Magnetic permeability constants: 


$$
\mu_{11}=297 \times 10^{-6} N s^{2} / C^{2}, \mu_{33}=83.5 \times 10^{-6} N s^{2} / C^{2}
$$

and all the absent material constants equal to zero. The dielectric constant and magnetic permeability of air or vacuum are, respectively, $\kappa_{0}=0.0885 \times 10^{-10} \mathrm{C}^{2} / \mathrm{Nm}^{2}$ and $\mu_{0}=1.256 \times 10^{-6} N s^{2} / C^{2}$.

\subsection{Effect of applied electric loads on crack tip field}

In Figs. 2 and 3 normalized stress intensity factor $K_{I} / K_{I 0}$ is plotted versus the applied electric loads $D_{2}^{\infty}$ for different dielectric constant and magnetic permeability of the crack interior, where $K_{I 0}=10 \sqrt{\pi a} \mathrm{MN} / \mathrm{m}^{3 / 2}$. The applied mechanical loads and magnetic induction are chosen to be $\sigma_{22}^{\infty}=10 \mathrm{MPa}$ and $B_{22}^{\infty}=100 \mathrm{~N} /(\mathrm{Am})$, respectively. It can be found that the value of $K_{I} / K_{I 0}$ obtained is smaller than that without considering the crack surfaces electrostatic tractions, and it increases firstly then decreases with the applied electric loads. For $D_{2}^{\infty}=0$ the stress on the crack surfaces emanates from the coupling feature between mechanical and electric field. The related electric field is compensated increasing $D_{2}^{\infty}$, which leads to a condition where $\sigma_{22}^{c}=0$. The electrostatic tractions $\sigma_{22}^{c}$ do not change its sign at further enhancement of $D_{2}^{\infty}$ due to the square relation in Eq. (10), and crack surfaces electrostatic tractions may be neglected for large applied mechanical and small electric displacement loads. $K_{I} / K_{I 0}$ is approximated very well using the magnetoelectric impermeable crack model when the values of $D_{2}^{\infty}$ varies from 0 to 0.002 under $\sigma_{22}^{\infty}=10 \mathrm{MPa}$ and $B_{22}^{\infty}=100 \mathrm{~N} /(\mathrm{Am})$. It can also be seen that crack tip stress intensity factor $K_{I} / K_{I 0}$ has been greatly influenced by the dielectric constant $\kappa_{c}$ and the magnetic permeability $\mu_{c}$ of medium filled inside the crack. Generally, $K_{I} / K_{I 0}$ increases with increasing the value of $\kappa_{c}$ and decrease with increasing the value of $\mu_{c}$. 
Figs. 4-7 show that the numerical results for normalized electric displacement intensity factor $K_{D} / K_{D 0}$ and normalized magnetic induction factor $K_{B} / K_{B 0}$ with applied electric loads $D_{2}^{\infty}$ for different $\kappa_{c}$ and $\mu_{c}$, where $K_{D 0}=0.01 \sqrt{\pi a} \mathrm{C} / \mathrm{m}^{3 / 2}$ and $K_{B 0}=0.01 \sqrt{\pi a} \mathrm{~N} /\left(\mathrm{Am}^{3 / 2}\right)$. It is noted that $K_{D} / K_{D 0}$ increases firstly then decreases with $D_{2}^{\infty}$ for the case of $\kappa_{c}=\kappa_{0}, B_{2}^{\infty}=100 \mathrm{~N} /(\mathrm{Am})$ and $\sigma_{22}^{\infty}=10 \mathrm{MPa}$, the possible reason is that the applied electric displacement plays an important role in crack tip $K_{D} / K_{D 0}$ when $D_{2}^{\infty}$ is small, and effect of crack surfaces electrostatic tractions $\sigma_{22}^{c}$ on $K_{D} / K_{D 0}$ gradually becomes dominant as $D_{2}^{\infty}$ increases. Furthermore, magnetic permeability of the medium inside the crack also makes a large impact on crack tip electric displacement and magnetic induction intensity factors, one can observe that both $K_{D} / K_{D 0}$ and $K_{B} / K_{B 0}$ increase with the decreasing value of $\mu_{c}$ from Figs. 5 and 7.

\subsection{Effect of applied magnetic loads on crack tip field}

Normalized stress intensity factor $K_{I} / K_{I 0}$ is plotted versus the applied magnetic induction $B_{2}^{\infty}$ for different dielectric constant and magnetic permeability of the crack interior from Figs. 8-13. Normalized factors $K_{I 0}, K_{D 0}$ and $K_{B 0}$ are set to be the same values in section 4.1. From Figs. 8 and 9 we can see that both $B_{2}^{\infty}$ and $\mu_{c}$ have a small contributions to the crack tip mode-I stress intensity factor $K_{I} / K_{I 0}$, however, the influence of $\kappa_{c}$ on $K_{I} / K_{I 0}$ is very large. It is also found that the effect of crack surfaces electrostatic tractions on $K_{D} / K_{D 0}$ and $K_{B} / K_{B 0}$ cannot be neglected when a large magnetic induction loads $B_{2}^{\infty}$ is applied, and both $K_{D} / K_{D 0}$ and $K_{B} / K_{B 0}$ increase with the increasing $B_{2}^{\infty}$ by considering the electrostatic tractions $\sigma_{22}^{c}$ from Figs. 10-13. Finally, it is important to note that the electrostatic tractions on the crack surfaces always have the tendency to close the crack thus retard the crack propagation, 
and the traditional traction-free crack model overestimates the effect of applied magnetoelectric loads on the crack tip filed intensity factors based on the numerical results obtained from Figs. 2-13.

\section{Conclusions}

The fracture mechanics problem of magnetoelectroelastic material subjected to remote mechanical, electric and magnetic loads is successfully studied by extending Stroh formalism and continuous distribution generalized dislocation method in this paper. Magnetoelectric semi-permeable crack model is adopted to investigate the effect of crack surfaces electrostatic tractions on crack tip fields. Explicit and closed-form expressions of electric displacement and magnetic induction inside the crack, and stress, electric displacement and magnetic induction intensity factors are obtained. Numerical results show that the crack surfaces electrostatic tractions should be considered for large applied magnetic or electric loads as well as small mechanical loads, dielectric constant and magnetic permeability of the crack interior.

Acknowledgements: The research was supported by the National Science Foundation of China (NSFC) (Project Nos. 11402063, 11172081 and 11372086), the Natural Science Foundation of Guangdong Province of China (2016A030311006), Research Innovation Fund of Shenzhen City of China (JCYJ20150805142729431), Zhejiang Provincial Top Key Discipline of Mechanics Open Foundation (xklx1604) and the K. C. Wong Magna Fund in Ningbo University.

\section{References}

Alshits, I., Darinskii, A.N., Lothe, J., 1992. On the existence of surface waves in 
half-anisotropic elastic media with piezoelectric and piezomagnetic properties. Wave Motion 16, 265-283.

Bhargava, R.R., Verma, P.R., Jangid, K., 2015. A mathematical strip-induction-saturation model for an off-centric semipermeable crack in a piezoelectromagnetic strip. Acta Mech. 226, 351-370.

Gao, C.F., Kessler, H., Balke, H., 2003. Crack problems in magnetoelectroelastic solids. Part I: exact solution of a crack. Int. J. Eng. Sci. 41, 969-981.

Gao, C.F., Tong, P., Zhang, T.Y., 2004. Fracture mechanics for a mode III crack in a magnetoelectroelastic solid. Int. J. Solids Struct. 41, 6613-6629.

García-Sánchez, F., Rojas-Díaz, R., Sáez, A., Zhang, Ch., 2007. Fracture of magnetoelectroelastic composite materials using boundary element method (BEM). Theor. Appl. Fract. Mech. 47, 192-204.

Hao, T.H., Shen, Z.Y., 1994. A new electric boundary condition of electric fracture mechanics and its applications. Eng. Fract. Mech. 47, 793-802.

Hu, K.Q., Chen, Z.T., Fu, J.W., 2014. Dynamic analysis of an interface crack between magnetoelectroelastic and functionally graded elastic layers under anti-plane mechanical and in-plane electro-magnetic loadings. Compos. Struct. 142-148.

Hu, K.Q., Chen, Z.T., Fu, J.W., 2015. Moving Dugdale crack along the interface of two dissimilar magneotoelectroelastic materials. Acta Mech. 226, 2065-2076.

Huang, J.H., Kuo, W.S., 1997. The analysis of piezoelectric/piezomagnetic composite materials containing ellipsoidal inclusions. J. Appl. Phys. 81, 1378-1386.

Landis, C.M., 2004. Energetically consistent boundary conditions for electromechanical fracture. Int. J. Solids Sturct. 41, 6291-6315.

Li, Q., Ricoeur, A., Kuna, M., 2011. Coulomb traction on a penny-shaped crack in a three dimensional piezoelectric body. Arch. Appl. Mech. 81, 685-700. 
Liu, J.X., Liu, X.L., Zhao, Y.B., 2001. Green's functions for anisotropic magnetoelectroelastic solids with an elliptical cavity or a crack. Int. J. Eng. Sci. $39,1405-1418$.

Ma, P., Feng, W.J., Su, R.K.L., 2013. Pre-fracture zone model on electrically impermeable and magnetically permeable interface crack between two dissimilar magnetoelectroelastic materials. Eng. Fract. Mech. 102, 310-323.

Ma, P., Su, R.K.L., Feng, W.J., 2014. Fracture analysis of an electrically conductive interface crack with a contact zone in a magnetoelectroelastic biomaterial system. Int. J. Solids Struct. 53, 48-57.

McMeeking, R.M., Landis, C.M., 2005. Electrostatic forces and stored energy for deformable dielectric materials. J. Appl. Mech. 72, 581-590.

Nan, C.W., 1994. Magnetoelectric effect in composites of piezoelectric and piezomagnetic phases. Phys. Rev. B 50, 6082-6088.

Nan, C.W., Bichurin, M.I., Dong, S.X, Viehland, D., Srinivasan, G., 2008. Multiferroic magnetoelectric composites: Historical perspective, status, and future directions. J. Appl. Phys. 103, 031101.

Neumeister, P., Jurisch, M., Jelitto, H., Engert, A.R., Schneider, G.A., Balke, H., 2013. Effective permittivity of air-filled cracks in piezoelectric ceramics due to crack bridging. Acta Mater. 61, 1061-1069.

Ootao, Y., Tanigawa, Y., 2005. Transient analysis of multilayered magneto-electro-thermoelastic strip due to nonuniform heat supply. Compos. Strcut. 68, 471-480.

Qin, Q.H., 2004. Green's functions of magnetoelectroelastic solids with a half-plane boundary or bimaterial interface. Phil. Mag. Lett. 84, 771-779.

Qin, Q.H., 2005. 2D Green's of defective magnetoelectroelastic solids under thermal 
loading. Eng. Anal. Bound. Elem. 29, 577-585.

Riceur, A., Kuna, M., 2009. Electrostatic tractions at crack faces and their influence on the fracture mechanics of piezoelectrics. Int. J. Fract. 157, 3-12.

Rogowski, B., 2015. The transient analysis of a conducting crack in magneto-electro-elastic half-space under anti-plane mechanical and in-plane electric and magnetic impacts. Arch. Appl. Mech. 85, 29-50.

Rojas-Díaz, R., Denda, M., García-Sánchez, F., Sáez, A., 2012. Dual BEM analysis of different crack face boundary conditions in 2D magnetoeletroelastic solids. Eur. J. Mech. A/Solids 31, 152-162.

Sih, G.C., Chen, E.P., 2003. Dilatational and distortional behavior of cracks in magnetoelectroelastic materials. Theor. Appl. Fract. Mech. 40, 1-21.

Sih, G.C., Song, Z.F., 2003. Magnetic and electric poling effects associated with crack growth in $\mathrm{BaTiO}_{3}-\mathrm{CoFe}_{2} \mathrm{O}_{4}$ composite. Theor. Appl. Fract. Mech. 39, 209-227.

Sladek, J., Sladek, V., Krahulec, S., Chen, C.S., Young, D.L., 2015. Analyses of circular magnetoelectroelastic plates with functionally graded material properties. Mech. Adv. Mater. Struct. 22, 479-489.

Soh, A.K., Liu, J.X., 2005. On the constitutive equations of magnetoelectroelastic solids. J. Intel. Mater. Syst. Str. 597-602.

Song, Z.F., Sih, G.C., 2003. Crack initiation behavior in magnetoelectroelastic composite under in-plane deformation. Theor. Appl. Fract. Mech. 39, 189-207.

Suo, Z., 1990. Singularities, interfaces and cracks in dissimilar anisotropic media. Proc. R. Soc. Lond. A 427, 331-358.

Tian, W.Y., Gabbert, U., 2004. Multiple crack interaction problem in magnetoelectroelastic solids. Eur. J. Mech. A/Solids 23, 599-614.

Tupholme, G.E., 2009. Moving antiplane shear crack in transversely isotropic 
magnetoelectroelastic media. Acta Mech. 202, 153-162.

Wang, B.L., Mai, Y.W., 2007. Applicability of the crack-face electromagnetic boundary conditions for fracture of magnetoelectroelastic materials. Int. J. Solids Struct. 44, 387-398.

Wang, X., Shen, Y.P., 2002. The general solution of three-dimensional problems in magnetoelectroelastic media. Int. J. Eng. Sci. 40, 1069-1080.

Xie, T., Fan, C.Y., Liu, H.T., Zhao, M.H., Zhang, T.Y., 2014. Effect of electrostatic tractions on the fracture behavior of a piezoelectric material under mechanical and/or electric loading. Theor. Appl. Fract. Mech. 69, 6-16.

Zhang, A.B., Wang, B.L., 2014b. The influence of Maxwell stresses on the fracture mechanics of piezoelectric materials. Mech. Mater. 68, 64-69.

Zhang, A.B., Wang, B.L., 2014a. Theoretical model of crack branching in magnetoelectric thermoelastic materials. Int. J. Solids Struct. 51, 1340-1349.

Zhong, X.C., Li, X.F., 2007. Magnetoelectroelastic analysis for an opening crack in a piezoelectromagnetic solid. Eur. J. Mech. A/Solids 26, 405-417. 


\section{List of figure captions}

Fig. 1 An opening crack model with the dielectric constant $\kappa_{c}$ and magnetic permeability $\mu_{c}$ of crack interior in a magnetoelectric composite material

Fig. 2 Normalized stress intensity factor $K_{I}$ verse applied electric loads $D_{2}^{\infty}$ with different dielectric constants $\kappa_{c}$ inside the crack

Fig. 3 Normalized stress intensity factor $K_{I}$ verse applied electric loads $D_{2}^{\infty}$ with different magnetic permeability $\mu_{c}$ inside the crack

Fig. 4 Normalized electric displacement intensity factor $K_{D}$ verse applied electric loads $D_{2}^{\infty}$ with different dielectric constants $\kappa_{c}$ inside the crack

Fig. 5 Normalized electric displacement intensity factor $K_{D}$ verse applied electric loads $D_{2}^{\infty}$ with different magnetic permeability $\mu_{c}$ inside the crack

Fig. 6 Normalized magnetic induction intensity factor $K_{B}$ verse applied electric loads $D_{2}^{\infty}$ with different dielectric constants $\kappa_{c}$ inside the crack

Fig. 7 Normalized magnetic induction intensity factor $K_{B}$ verse applied electric loads $D_{2}^{\infty}$ with different magnetic permeability $\mu_{c}$ inside the crack

Fig. 8 Normalized stress intensity factor $K_{I}$ verse applied magnetic loads $B_{2}^{\infty}$ with different dielectric constants $\kappa_{c}$ inside the crack

Fig. 9 Normalized stress intensity factor $K_{I}$ verse applied magnetic loads $B_{2}^{\infty}$ with different magnetic permeability $\mu_{c}$ inside the crack

Fig. 10 Normalized electric displacement intensity factor $K_{D}$ verse applied magnetic loads $B_{2}^{\infty}$ with different dielectric constants $\kappa_{c}$ inside the crack 
Fig.11 Normalized electric displacement intensity factor $K_{D}$ verse applied magnetic loads $B_{2}^{\infty}$ with different magnetic permeability $\mu_{c}$ inside the crack

Fig. 12 Normalized magnetic induction intensity factor $K_{B}$ verse applied magnetic loads $B_{2}^{\infty}$ with different dielectric constants $\kappa_{c}$ inside the crack

Fig. 13 Normalized magnetic induction intensity factor $K_{B}$ verse applied magnetic loads $B_{2}^{\infty}$ with different magnetic permeability $\mu_{c}$ inside the crack 

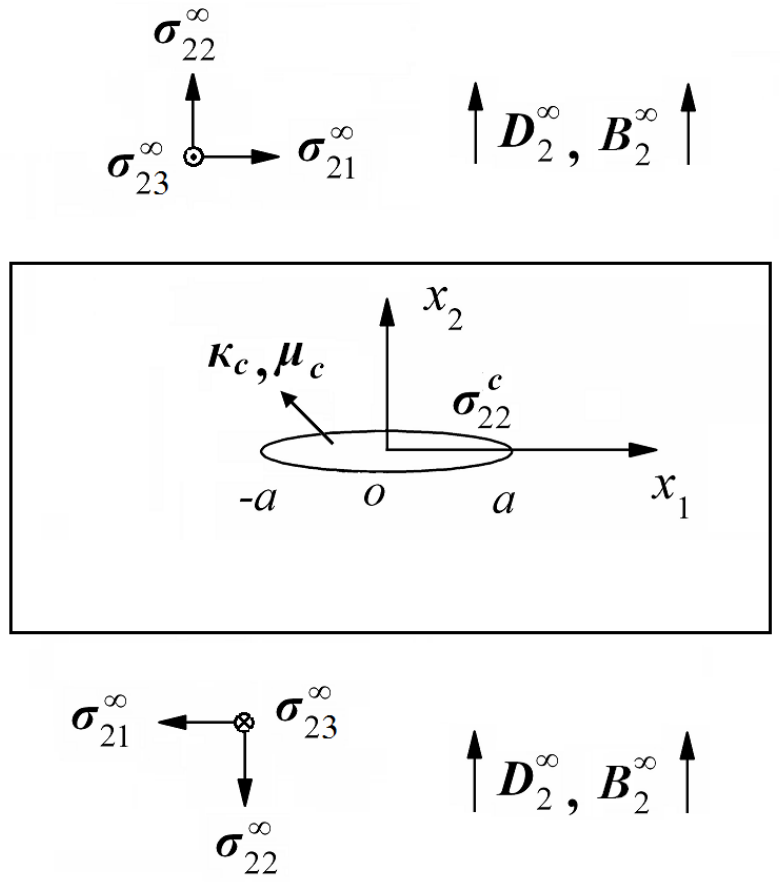

Fig. 1 


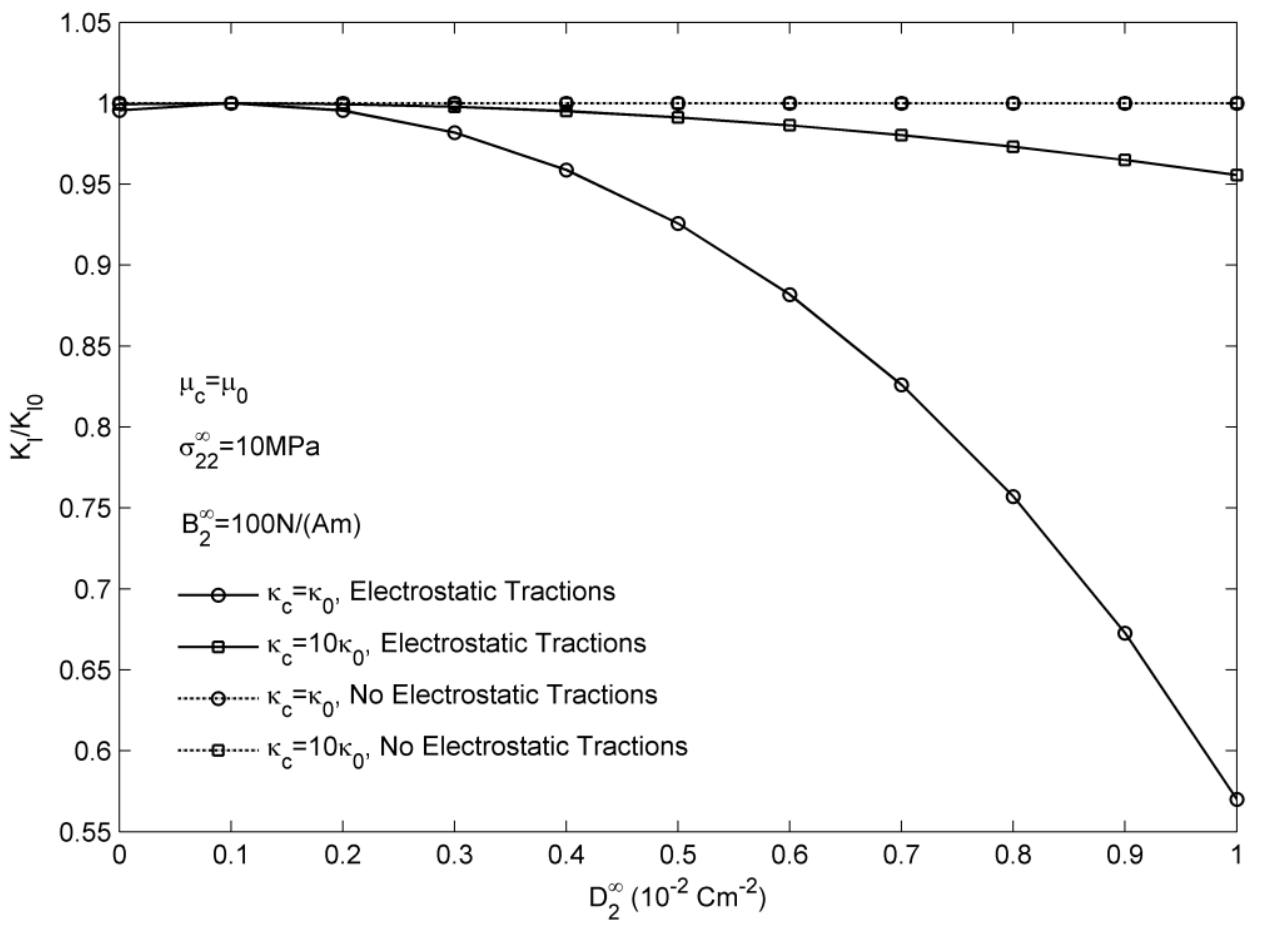

Fig. 2 


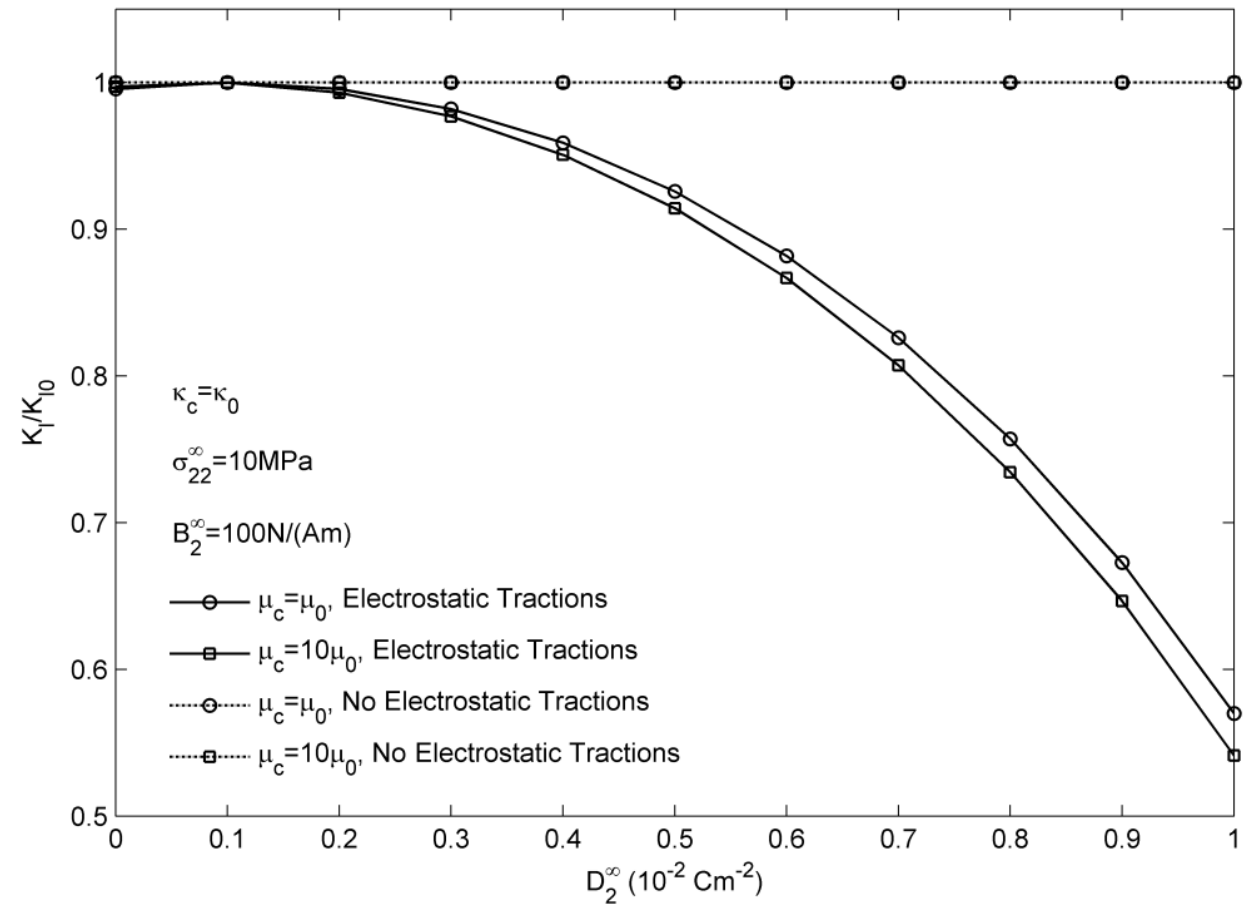

Fig. 3 


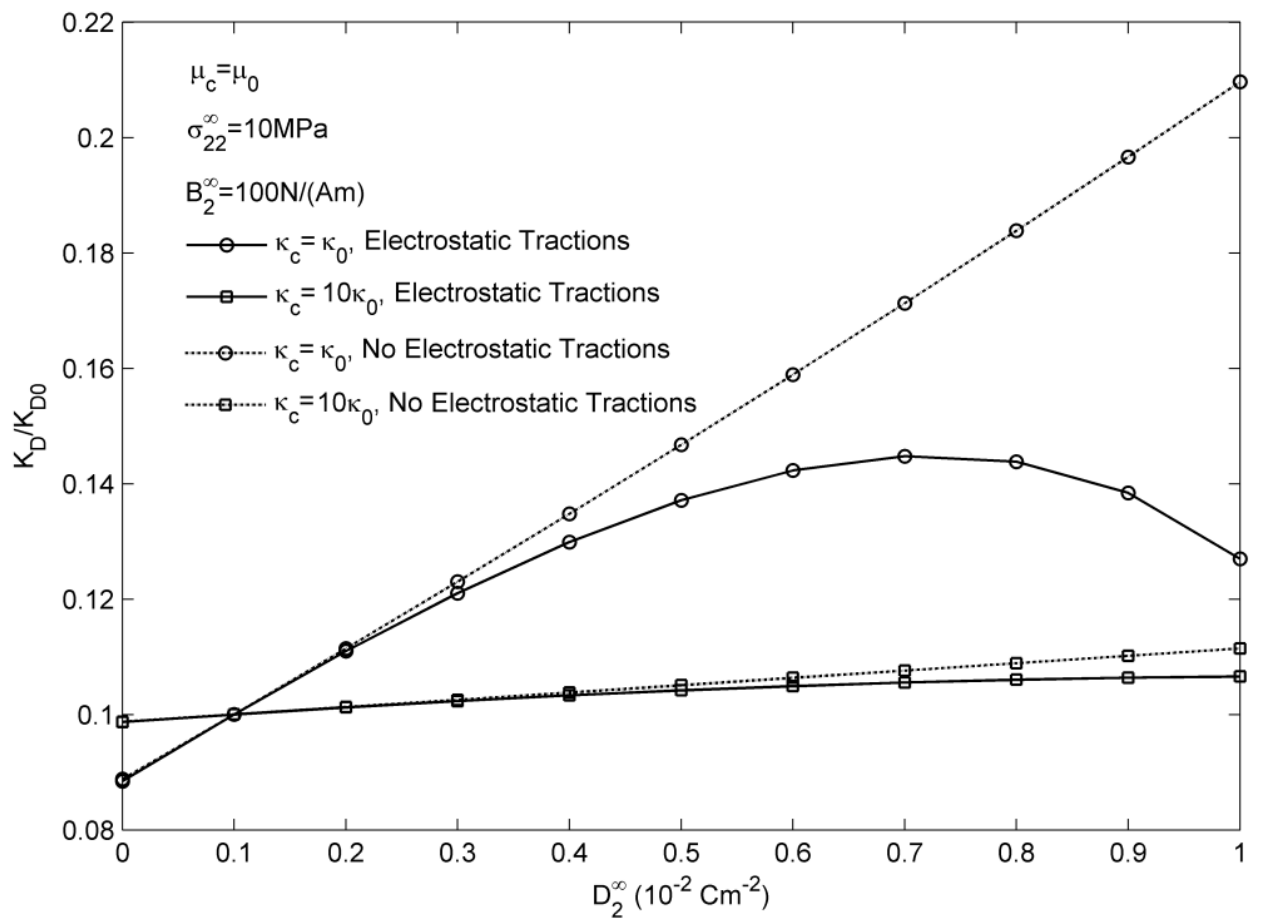

Fig. 4 


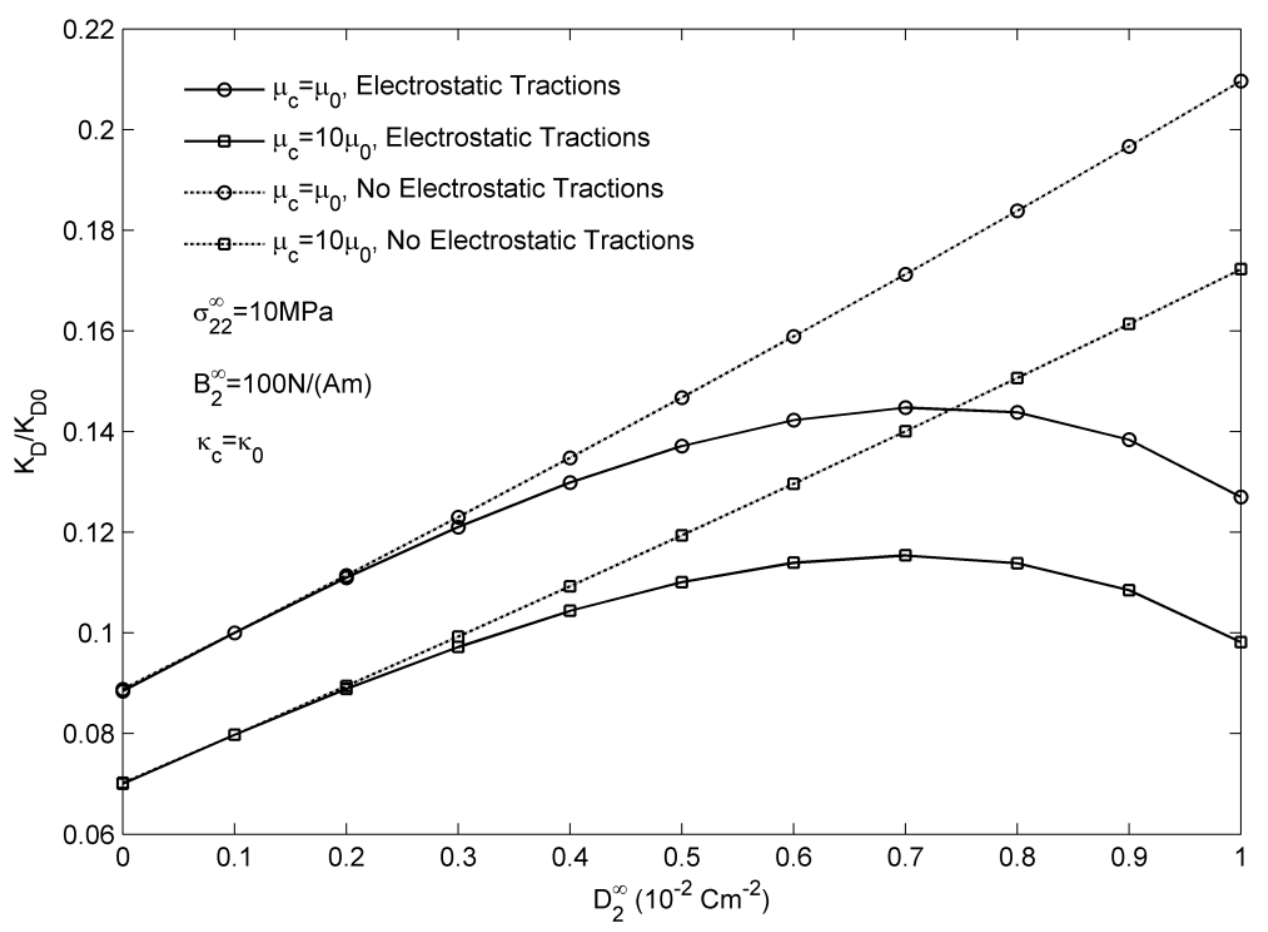

Fig. 5 


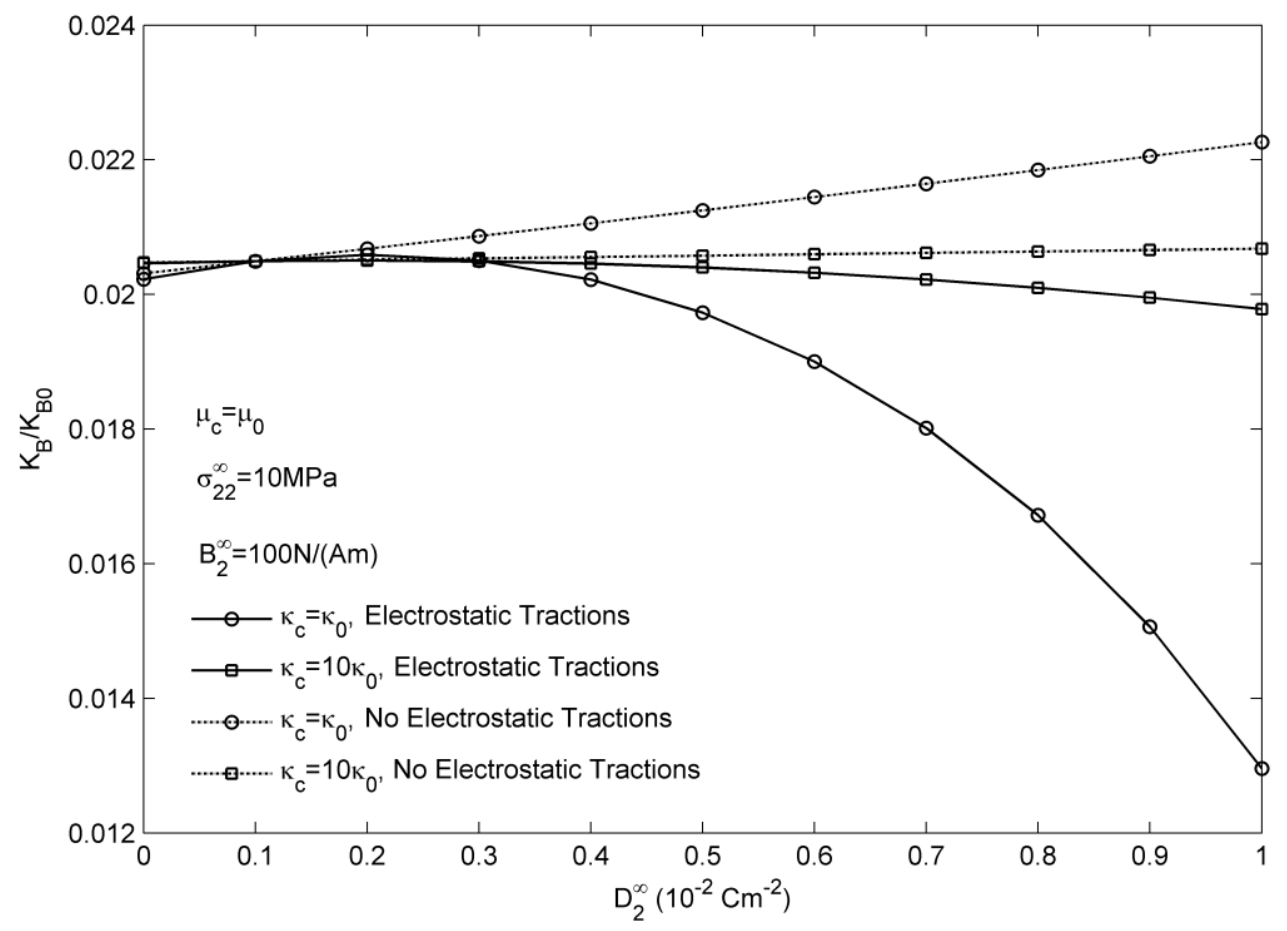

Fig. 6 


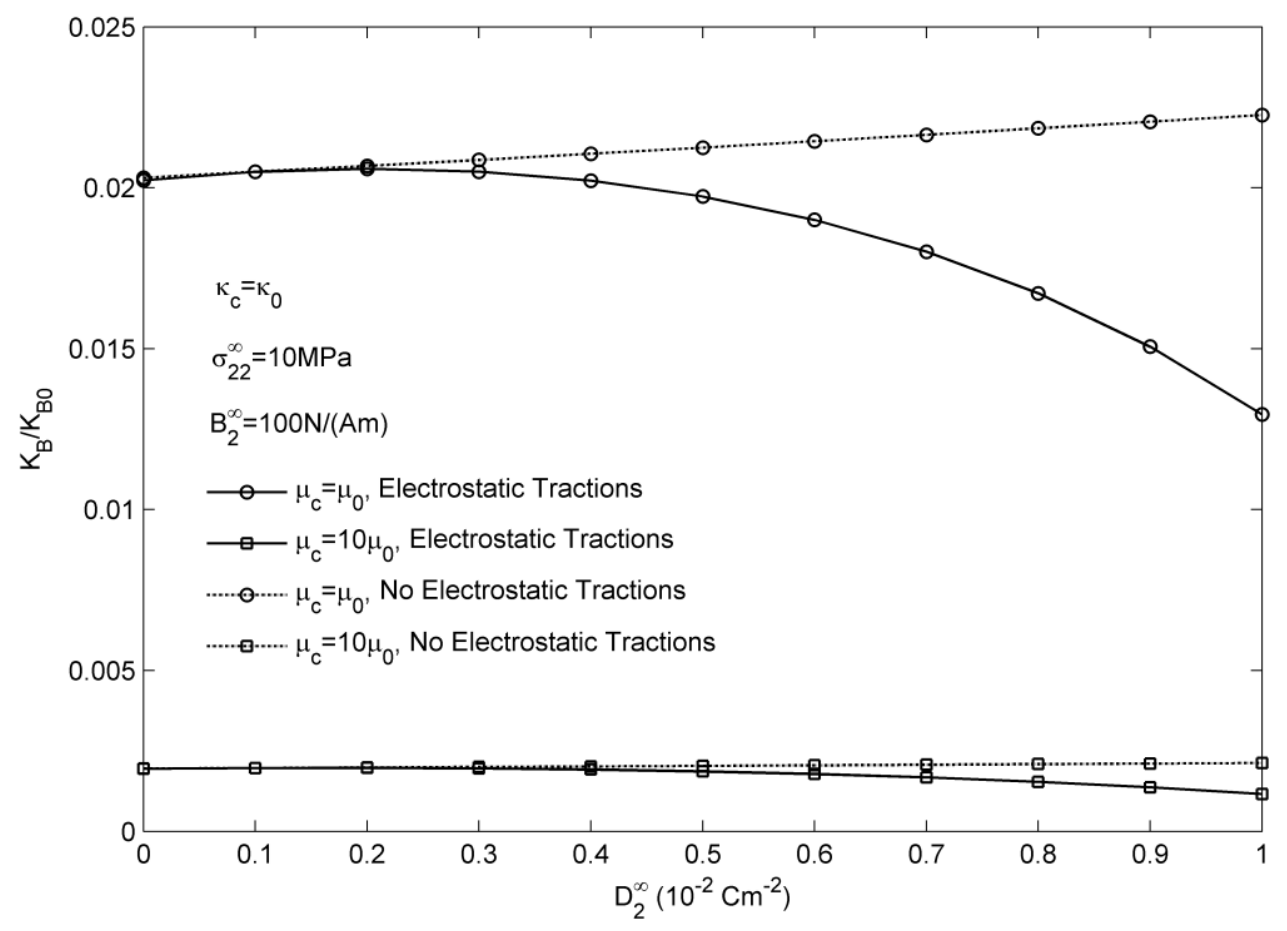

Fig. 7 


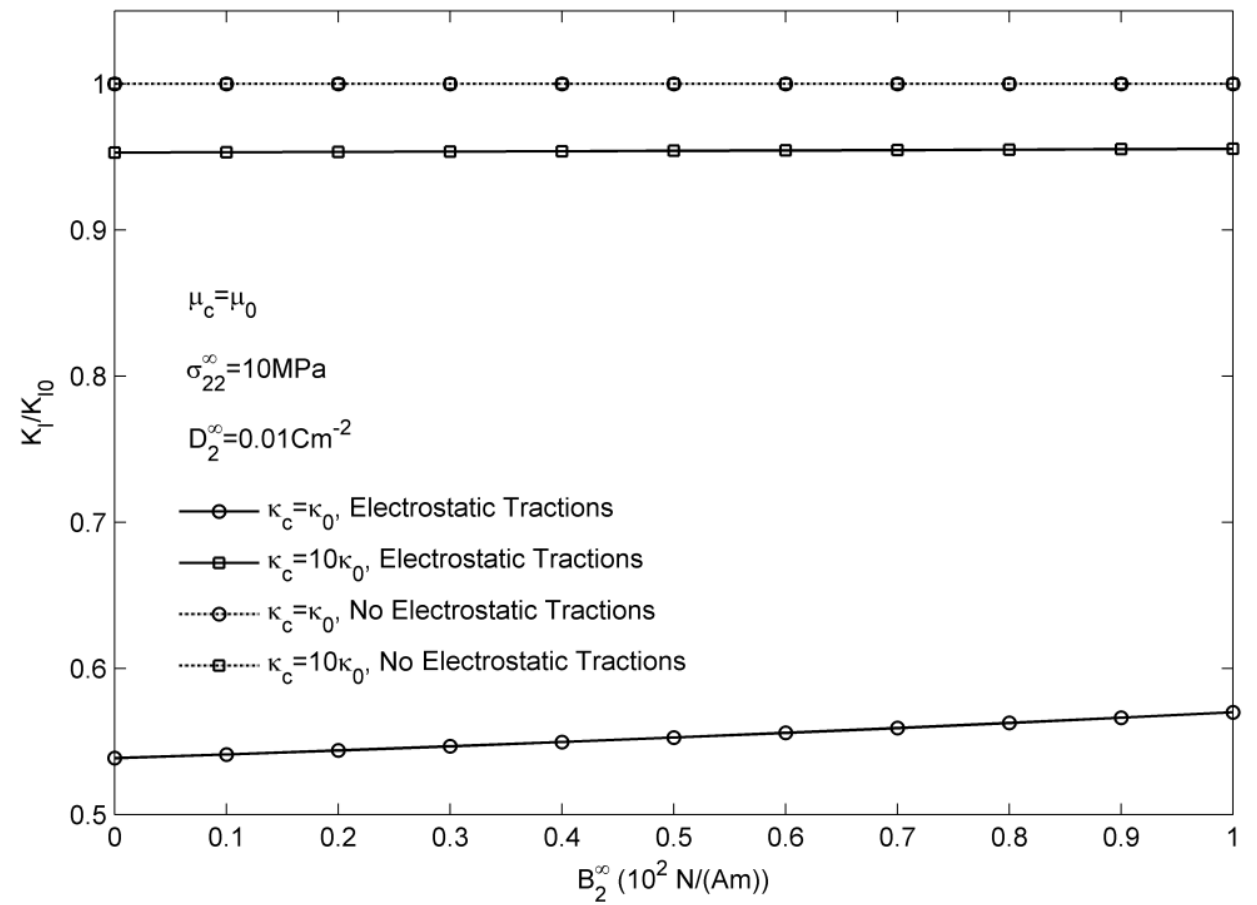

Fig. 8 


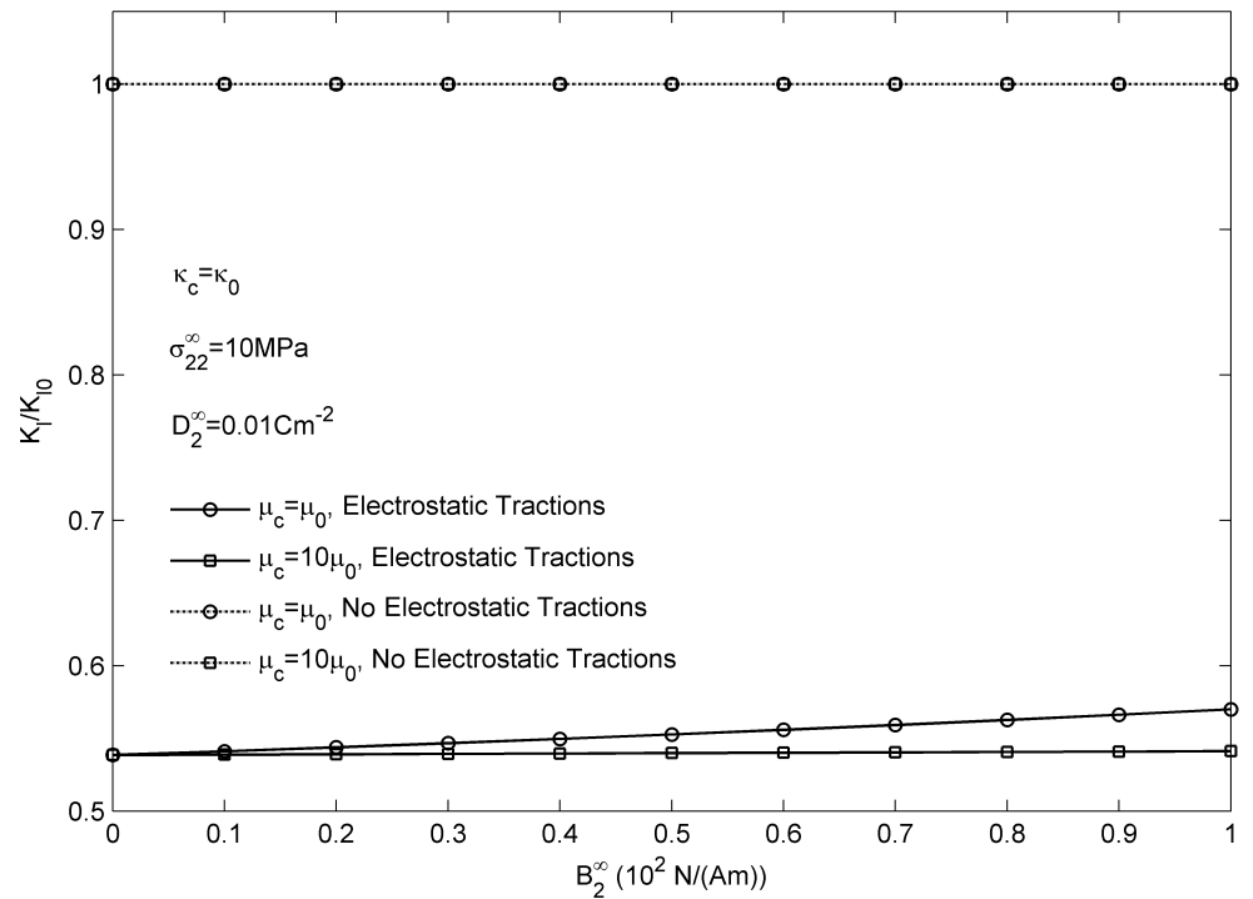

Fig. 9 


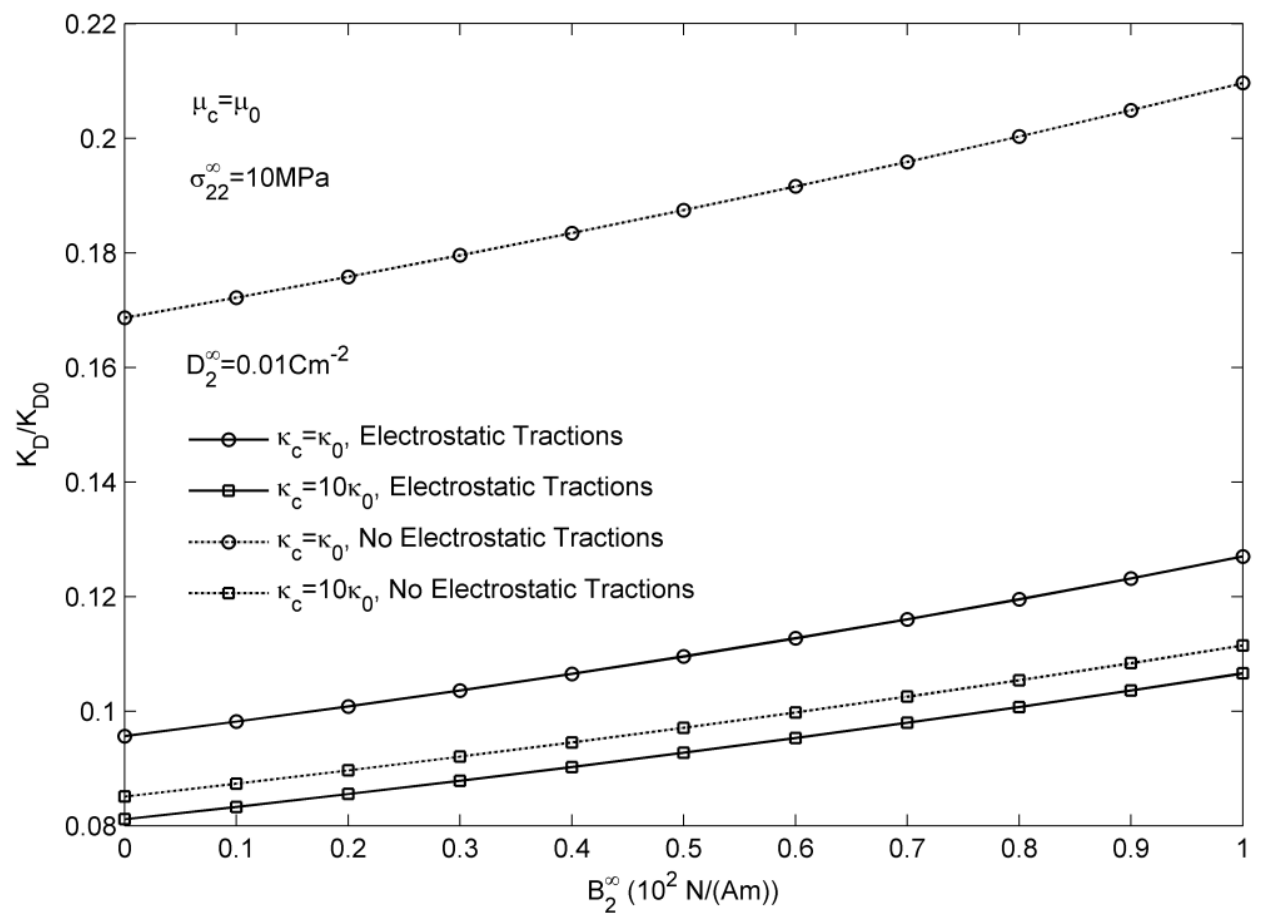

Fig. 10 


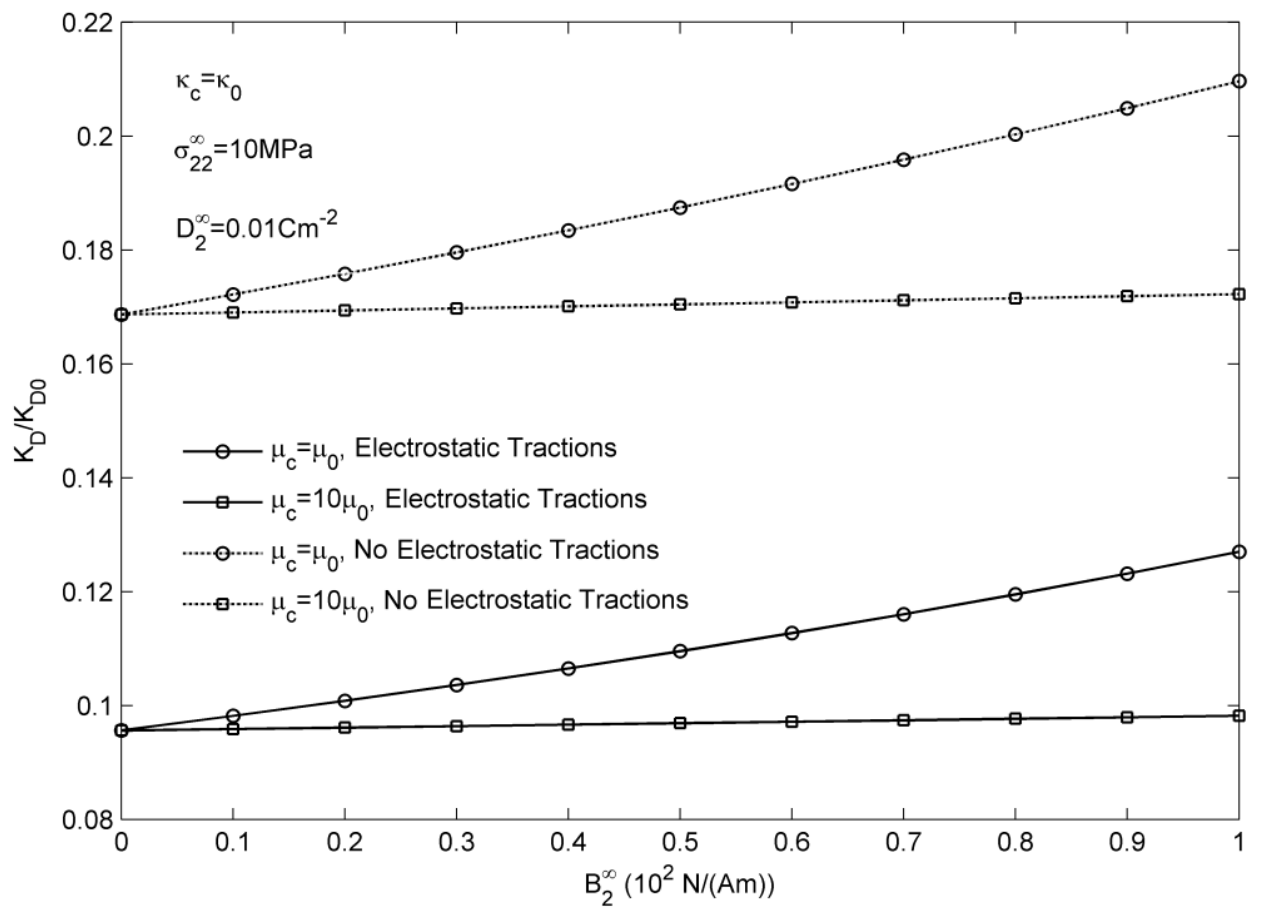

Fig. 11 


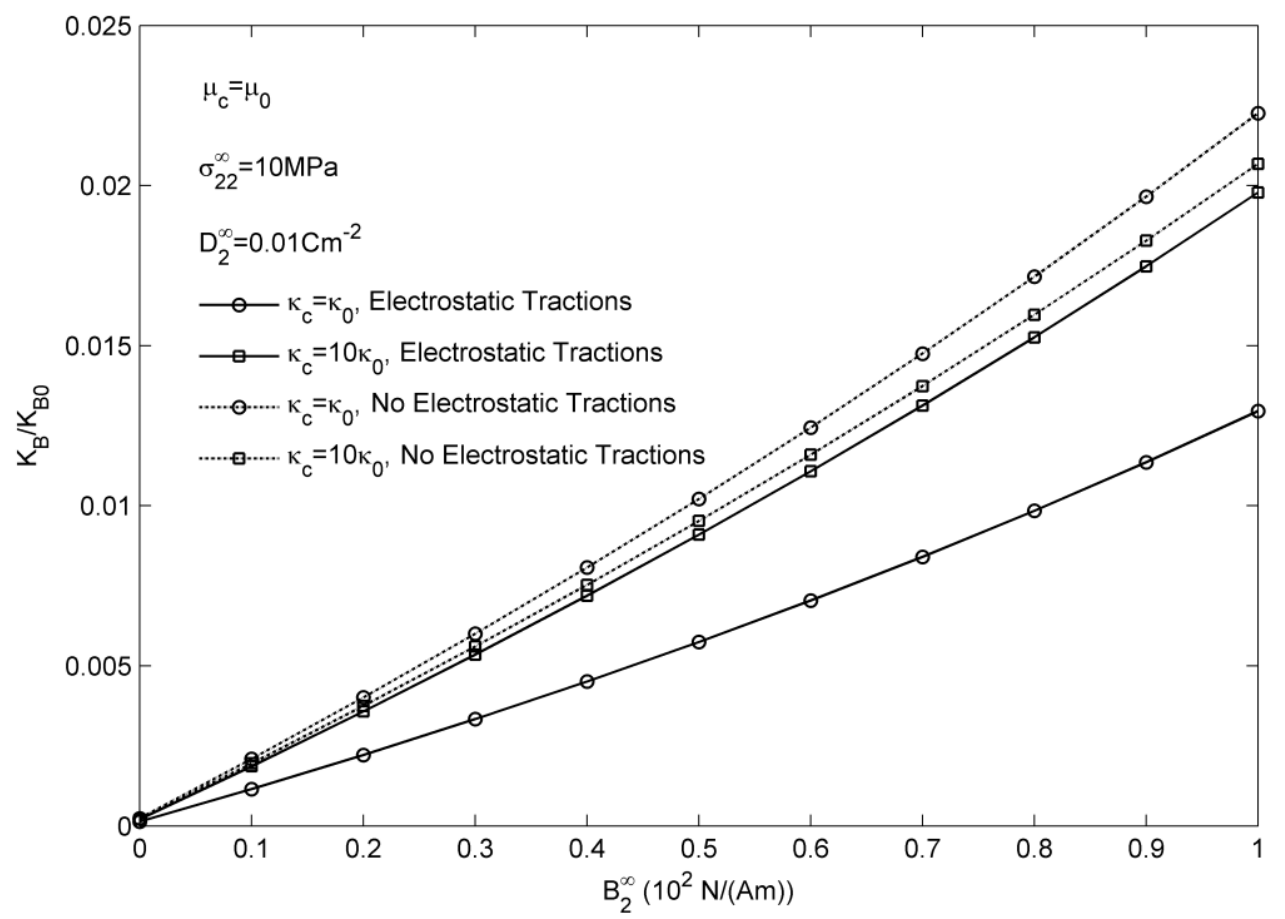

Fig. 12 


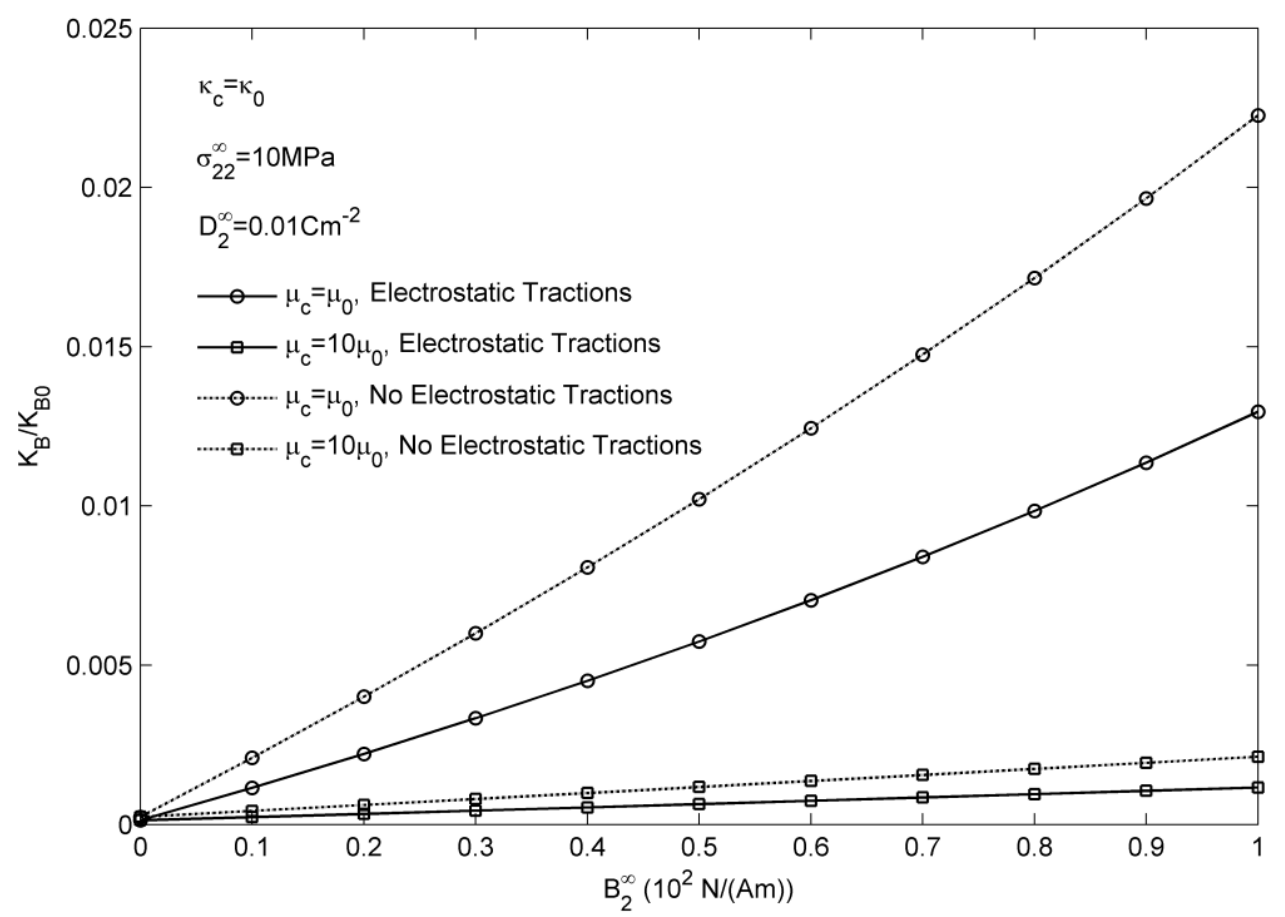

Fig. 13 